\title{
THE CONFORMAL STRUCTURE AND GEOMETRY OF TRIPLY PERIODIC MINIMAL SURFACES IN $\mathbf{R}^{3}$
}

\author{
BY WILLIAM H. MEEKS, III ${ }^{1}$ \\ Communicated by M. A. Rosenlicht, September 1, 1976
}

\begin{abstract}
Working within the conformal category, we develop complementary existence and rigidity theories for periodic minimal surfaces in $\mathrm{R}^{n}$.
\end{abstract}

We will call a compact Riemann surface $M$ periodic if it conformally minimally immerses in a flat three-torus $T^{3}$. By lifting to the universal cover of $T^{3}$, these periodic surfaces become the proper triply periodic minimal surfaces in $\mathbf{R}^{\mathbf{3}}$.

We find that the compactness of a minimal surface $M$ in $T^{3}$ gives rise to restrictions on the conformal type of $M$. Frequently, these conformal restrictions give nontrivial geometric information about the lifted minimal surface in $\mathbf{R}^{\mathbf{3}}$. For this reason, we consider the following fundamental problems:

(1) Which compact Riemann surfaces are periodic?

(2) How does the conformal structure of a periodic surface influence its geometry?

Our first result on these questions is that a surface of genus two is never periodic. Since every surface of genus two is hyperelliptic, this follows from our more general result that a hyperelliptic Riemann surface of even genus is never periodic. We also find another family of nonperiodic surfaces: Any nonsingular curve of degree four in $C P^{2}$ fails to be periodic. Thus, the classical Fermat curve of degree four in $C P^{2}$ given in homogeneous coordinates by $x^{4}+y^{4}+z^{4}=0$ provides a good example of a nonperiodic surface. The techniques of proof used here consist of a study of the Gauss map of a minimal surface and the canonical curve of a Riemann surface.

Besides finding conformal obstructions to periodicity, we also begin the development of a general existence theory. Much of this existence theory is based on our rigidity theorems for periodic and nonperiodic minimal surfaces in $\mathbf{R}^{\mathbf{3}}$ and on the study of the canonical curve of a Riemann surface. One consequence of joining these theories is that we can show the Schwartz diamond surface can be joined to its conjugate surface through minimal surfaces in flat three-tori.

The following is a list of our basic results.

THEOREM 1. There exists a real 5-dimensional family $V$ of periodic hyperelliptic surfaces of genus 3. The surfaces in $V$ are the two-sheeted covers of $S^{2}$ branched over 8 antipodal points.

AMS (MOS) subject classifications (1970). Primary 53A10.

${ }^{1}$ The author was supported by NSF grant MPS71-02597.

Copyright $\odot 1977$, American Mathematical Society 
Since the minimal surfaces in the family $V$ are embeddings, the following result takes on a particular significance.

THEOREM 2. If $f: M \rightarrow T^{3}$ is a minimal surface of genus 3 , then

(1) $M$ is hyperelliptic,

(2) there exist 8 zeroes of Gauss curvature,

(3) the hyperelliptic automorphism is an isometry and induces an inversion symmetry through each zero of Gauss curvature,

(4) if $f: M \rightarrow T^{3}$ is an embedding, then after translation, the zeroes of Gauss curvature are the order 2 points of $T^{3}$.

Every compact Riemann surface of genus $g$ has a conformal minimal embedding in a flat $2 g$-torus which is its Jacobian. Our next result improves this property by one dimension.

THEOREM 3. (1) If the genus $g$ of $M$ is greater than 3, then $M$ will conformally minimally immerse fully in a flat $2 g-1$ torus.

(2) A surface of genus 3 will conformally minimally immerse fully in a flat 5-torus if and only if the surface is hyperelliptic.

The geometric conditions in the next theorem hold for several classical examples. By this theorem, these examples give rise to an infinite number of distinct isometric periodic minimal surfaces.

THEOREM 4. If $f: M \rightarrow T^{3}=\mathbf{R}^{3} / Z^{3}$ is a minimal surface with the "symmetries of a cube" and the conjugate surface is a closed subset of $\mathbf{R}^{3}$, then for a dense set of angles $\theta \in S^{1}$, the associate surfaces induce isometric minimal immersions $f_{\theta}: M \rightarrow T_{\theta}^{3}$. Also, the Jacobian of $M$ is isogeneous to a product $T_{\mathbf{C}}^{1} \times$ $T_{\mathrm{C}}^{1} \times T_{\mathrm{C}}^{1} \times T_{\mathrm{C}}^{g-3}$ where $T_{\mathrm{C}}^{1}$ is a rectangular elliptic curve and $g$ is the genus of $M$.

Rigidity Theorem 5. (1) Proper triply periodic minimal surfaces in $\mathbf{R}^{\mathbf{3}}$ are rigid.

(2) Compact minimal surfaces in flat 3-tori are not rigid. In fact, there exists an isometry of order 8 on the Schwarz crossed layers of parallels surface that does not extend to an isometry of its 3-torus.

Finiteness THEOREM 6. The number of noncongruent isometric minimal immersions of a compact Riemannian manifold into a fixed flat torus is finite.

By combining geometric arguments with classification theorems of Papakyriakopoulos and Waldhausen we get the following "unknottedness" result for embedded periodic minimal surfaces in $\mathbf{R}^{\mathbf{3}}$.

THEOREM 7. If the genus of $M$ is greater than 1 and $f: M \rightarrow T^{3}$ is a miminal embedding, then $M$ disconnects $T^{3}$ into two g-holed handlebodies. Thus, an embedded periodic minimal surface in $\mathbf{R}^{\mathbf{3}}$ disconnects $\mathbf{R}^{3}$ into two diffeomorphic regions. 
Abel-Gauss-Bonnet Theorem for Periodic surfaces. If $f: M \rightarrow T^{3}$ is a minimal immersion of a surface of genus $g$, then the Gauss map $G: M \rightarrow S^{2}$ is a holomorphic $g-1$ branched cover of $S^{2}$ and $\sum_{g \in G^{-1}(P)} q \in T^{3}$ is independent of $P \in S^{2}$.

\section{REFERENCES}

1. H. B. Lawson, Jr., Lectures on minimal submanifolds, I. M. P. A., Rua Luiz de Camōes 68, Rio de Janeiro, 1973.

2. Complete minimal surfaces in $s^{3}$, Ann. of Math. (2), 92 (1970), 335-374. MR 42 \# 5170.

3. W. Meeks, Thesis, Univ. of California, Berkeley, 1975.

4. A. Schoen, Infinite periodic minimal surfaces without self-intersections, NASA Technical Note, TN D-5541, 1970.

DEPARTMENT OF MATHEMATICS, UNIVERSITY OF CALIFORNIA, LOS ANGELES, CALIFORNIA 90024 